\title{
The Complexity of Measuring Power in Generalized Opinion Leader Decision Models
}

\author{
Xavier Molinero ${ }^{1,3}$ \\ Department of Mathematics. \\ Technical University of Catalonia. \\ Manresa, Spain \\ Maria Serna ${ }^{2,4}$ \\ Computer Science Department. \\ Technical University of Catalonia. \\ Barcelona, Spain
}

\begin{abstract}
We analyze the computational complexity of the power measure in models of collective decision: the generalized opinion leader-follower model and the oblivious and non-oblivious influence models. We show that computing the power measure is \#Phard in all these models, and provide two subfamilies in which the power measure can be computed in polynomial time.

Keywords: Decision-making models, Power measure, Computational complexity

1 Partially funded by Grant MTM2015-66818-P from MINECO.

2 Partially funded by MINECO and FEDER funds under grant TIN2013-46181-C2-1-R, and AGAUR grant SGR 2014-1034.

3 Email: xavier.molinero@upc.edu

${ }^{4}$ Email: mjserna@cs.upc.edu
\end{abstract}




\section{Introduction}

Opinion leadership is a well known and established model for communication in sociology and marketing. It comes from the two-step flow of communication theory proposed in the 1940s [1]. This theory recognizes the existence of collective decision-making situations in societies formed by actors called opinion leaders.Following those ideas an opinion leader-follower model (OLF) was introduced in [4] together with two measures associated to participants satisfaction (SAT) and power (Pow). Cooperative influence games were introduced in [3] based on how influence is exerted in the linear threshold model. Decision models extending OLF to general influence graphs: generalized opinion leader-follower model (gOLF), and oblivious and non-oblivious influence decision models, were introduced in [2] together with an analysis of the complexity of computing the SAT measure. Here we analyze the computational complexity of the Pow measure showing that it presents the same behavior as SAт: computing Pow is \#P-hard, for the decision models considered in this paper, while it can be computed in polynomial time in strong hierarchical and star influence graphs.

\section{Decision models}

All the graphs considered in this paper are directed, without loops and multiple edges. We use standard notation: $G=(V, E)$ is a directed graph, $V(G)$ denotes the vertex set, $E(G)$ is the edge set, and $n$ denotes the number of vertices $|V|$. We use simply $V$ and $E$ when there is no risk of confusion. For $i \in V, S_{G}(i)=\{j \in V \mid(i, j) \in E\}$ denotes the set of successors of $i$, and $P_{G}(i)=\{j \in V \mid(j, i) \in E\}$ the set of predecessors of $i$. A two layered bipartite graph is a bipartite graph $G=\left(V_{1}, V_{2}, E\right)$ with $V(G)=V_{1} \cup V_{2}$ and $E \subseteq V_{1} \times V_{2}$, i.e., so that for $i \in V_{1}, P_{G}(i)=\emptyset$ and, for $i \in V_{2}, S_{G}(i)=\emptyset$. By $I_{a}$, as usual, we denote a graph that is formed by $a$ isolated vertices. Given $G=(V, E)$ and $X \subseteq V, G[X]$ denotes the subgraph induced by $X$ and $G \backslash X$ denotes the subgraph induced by $V \backslash X$, i.e., $G \backslash X=G[V \backslash X]$. For an acyclic graph $G$, I denotes the set of vertices with $P_{G}(i)=\emptyset$ and $S_{G}(i)=\emptyset$; L those with $P_{G}(i)=\emptyset$ and $S_{G}(i) \neq \emptyset$; F those with $P_{G}(i) \neq \emptyset$ and $S_{G}(i)=\emptyset$; and $\mathrm{FI}=\mathrm{F} \cup \mathrm{I}$. For a vector $x \in\{0,1\}^{n}$, let $X(x)=\left\{i \mid x_{i}=1\right\}$ and define $\bar{x}_{-i}$ as the vector that is $x$ but changing the component $x_{i}$ by $\overline{x_{i}}$.

Definition 2.1 A decision model $\mathcal{M}$ is a tuple $(V, D, q)$ where $V=\{1, \ldots, n\}$ and $D:\{0,1\}^{n} \rightarrow\{0,1\}^{n}$ is a function, and $0 \leq q \leq n+1$. For a participants' initial decision vector $x \in\{0,1\}^{n}$, the final decision vector is $y=D(x)$. The 
associated collective decision function $C_{\mathcal{M}}:\{0,1\}^{n} \rightarrow\{0,1\}$ is defined as $C_{\mathcal{M}}(x)=1$ iff $\left|\left\{i \in V \mid y_{i}=1\right\}\right| \geq q$.

Now we present the formal definition of opinion leader-follower models.

Definition 2.2 A generalized opinion leader-follower model (gOLF) is a defined by a triple $(G, r, q)$ where $G=(V, E)$ is a two layered bipartite digraph representing the actors' relations, $r$ is a rational number, $1 / 2 \leq r \leq 1$, and $q$ is an integer, $0<q \leq n$, defining a decision model $\mathcal{M}(G, r, q)=(V(G), D, q)$. The function $D$ is defined as follows, for $x \in\{0,1\}^{n}$, let $y=D(x)$, for $1 \leq i \leq n$, if for one $z \in\{0,1\},\left|\left\{j \in P_{G}(i) \mid x_{j}=z\right\}\right| \geq\left\lceil r \cdot\left|P_{G}(i)\right|\right\rceil$ and $\left|\left\{j \in P_{G}(i) \mid x_{j}=1-z\right\}\right|<\left\lceil r \cdot\left|P_{G}(i)\right|\right\rceil$, we set $y_{i}=z$ otherwise $y_{i}=x_{i}$.

An $O L F$, is a gOLF where $n$ is odd and $q=(n+1) / 2$. An odd-OLF is a gOLF $\mathcal{M}=(G, r, q)$ in which $r=1 / 2$ and, for all $i \in V$ with $P_{G}(i) \neq \emptyset$, $\left|P_{G}(i)\right|$ is odd.

Before defining influence based models, we recall the definitions of influence graphs and spread of influence from [3]. An influence graph is a tuple $(G, f)$, where $G=(V, E)$ is a directed graph and $f$ is a labeling function assigning to any vertex a non-negative rational value. Let $(G, f)$ be an influence graph and let $X \subseteq V$. The activation process, with initial activation $X$, at time $t$, $0 \leq t \leq n$, activates a set of vertices $F^{t}(X)$ defined as follows: $F^{0}(X)=X$ and $F^{t}(X)=F^{t-1}(X) \cup\left\{i \in V|| P_{G}(i) \cap F^{t-1}(X) \mid \geq f(i)\right\}$, for $1 \leq t \leq n$. The spread of influence of $X$ in $(G, f)$ is the set $F(X)=F^{n}(X)$.

Definition 2.3 An oblivious influence model is a decision model described by $(G, f, q, N)$, where $(G, f, q, N)$ is an influence graph with positive labeling function defining the model $\mathcal{M}(G, f, q, N)=(V(G), D, q)$ where , for $x \in$ $\{0,1\}^{n}, y=D(x)$ is defined as $y_{i}=1$ iff $i \in F(X(x) \cap N)$.

A non-oblivious influence model is a decision model described by $\mathcal{M}=$ $(G, f, q, N)$ where $(G, f, q, N)$ is an influence graph with positive labeling function defining the model $(V(G), D, q)$ where $y=D(x)$ is defined as follows. For $x \in\{0,1\}^{n}$, let $p_{i}^{1}(x)=|F(X(x) \cap N) \cap P(i)|$ and $p_{i}^{0}(x)=|P(i) \backslash F(X(x) \cap N)|$. For $i \in V(G) \backslash N, y_{i}=1$ iff $i \in F(X(x)$. For $i \in N$, if for one $z \in\{0,1\}$, $p_{i}^{z}(x) \geq f(i)$ and $p_{i}^{\bar{z}}(x)<f(i)$, we set $y_{i}=z$ otherwise $y_{i}=x_{i}$.

In general, a gOLF cannot be cast as an oblivious influence model because the tie-breaking rules are different. However odd-OLF constitute a submodel of both oblivious and non-oblivious influence models as ties do not arise.

Now we define the power measure introduced in [4].

Definition 2.4 Let $\mathcal{M}=(V, D, q)$ be a decision model. The power of $i \in V$, 
$\operatorname{Pow}_{\mathcal{M}}(i)$ is defined as $\left|\left\{x \in\{0,1\}^{n} \mid C(x)=x_{i} \wedge C\left(\bar{x}_{-i}\right)=\overline{C(x)}\right\}\right|$.

Associated with this measure we consider the Power problem: Given a decision model $\mathcal{M}$ and an actor $i$ compute $\operatorname{Pow}_{\mathcal{M}}(i)$.

\section{Complexity of computing Power}

Let $(G, f)$ be an influence graph, for $i \in V(G)$. For $N \subseteq V(G)$ and $1 \leq k \leq n$, $F_{k}(N, G, f)$ denotes the set $\{X \subseteq V(G)|| F(X \cap N) \mid=k\}$. The Expansion problem asks to compute $\left|F_{k}(N, G, f)\right|$, given an influence graph $(G, f), N \subseteq$ $V(G)$ and an integer $k$. The Expansion problem is known to be \#P-hard for odd-OLF taking $N=\mathrm{L} \cup \mathrm{I}[2]$.

Theorem 3.1 The Power problem is \#P-hard for odd-OLF models.

Proof. [Sketch] To show hardness we provide a reduction from the ExPAnsion on odd-OLF. Let $(G, f, N, k)$ be an input to the Expansion problem were $(G, f)$ is the graph corresponding to an odd-OLF $(G, r)$ and $N=\mathrm{L} \cup \mathrm{I}$. Consider the odd-OLF $\left(G^{\prime}, r\right)$ where $G^{\prime}$ is obtained from $G$ by adding an isolated new vertex $z$. Observe that $\left(G^{\prime}, r\right)$ is and odd-OLF with an additional independent participant. It is easy to see that for $\mathcal{M}=\mathcal{M}\left(G^{\prime}, r, k+1\right)$, $\operatorname{Pow}_{\mathcal{M}}(z)=\left|F_{k}(G, f, N)\right|$. Using this fact as the construction can be computed in polynomial time the results follows.

As an odd-OLF is a gOLF and also an oblivious and a non-oblivious influence model we get the following result.

Corollary 3.2 The Power problem for gOLF and oblivious and non-oblivious influence models is \#P-hard.

In the following we consider two subfamilies of bipartite digraphs, the strong hierarchical and the star influence graphs introduced in [2]. We devise polynomial time algorithms to solve Power for those families of graphs.

Strong hierarchical graphs are defined through some basic operations. Given two graphs $H_{1}$ and $H_{2}$ with $V\left(H_{1}\right) \cap V\left(H_{2}\right)=\emptyset$ their disjoint union is the graph $H_{1}+H_{2}=\left(V\left(H_{1}\right) \cup V\left(H_{2}\right), E\left(H_{1}\right) \cup E\left(H_{2}\right)\right)$. Given a graph $H$, the one layer extension to a set $V^{\prime} \neq \emptyset$ of new vertices $\left(V(H) \cap V^{\prime}=\emptyset\right)$ is the graph $H \otimes V^{\prime}$ is defines as $V\left(H \otimes V^{\prime}\right)=V(H) \cup V^{\prime}$ and $E\left(H \otimes V^{\prime}\right)=$ $E(H) \cup\left\{(u, v) \mid u \in \mathrm{FI}(H), v \in V^{\prime}\right\}$.

Observe that we have $\mathrm{L}\left(H_{1}+H_{2}\right)=\mathrm{L}\left(H_{1}\right) \cup \mathrm{L}\left(H_{2}\right), \mathrm{I}\left(H_{1}+H_{2}\right)=\mathrm{I}\left(H_{1}\right) \cup$ $\mathrm{I}\left(H_{2}\right), \mathrm{F}\left(H_{1}+H_{2}\right)=\mathrm{F}\left(H_{1}\right) \cup \mathrm{F}\left(H_{2}\right)$ and $\mathrm{FI}\left(H_{1}+H_{2}\right)=\mathrm{FI}\left(H_{1}\right) \cup \mathrm{FI}\left(H_{2}\right)$. 
Furthermore, $\mathrm{L}\left(H \otimes V^{\prime}\right)=\mathrm{L}(H) \cup \mathrm{I}(H), \mathrm{I}\left(H \otimes V^{\prime}\right)=\emptyset$, and $\mathrm{F}\left(H \otimes V^{\prime}\right)=$ $\mathrm{FI}\left(H \otimes V^{\prime}\right)=V^{\prime}$.

As base case we use graphs with only isolated vertices. The family is completed by taking the closure under the two graph operations defined above.

Definition 3.3 The family of strong hierarchical graphs is defined recursively as follows: (1) The graph $I_{a}$, for $a>0$, is a strong hierarchical graph; (2) if $H_{1}$ and $H_{2}$ are disjoint strong hierarchical graphs, the graph $H_{1}+H_{2}$ is a strong hierarchical graph; and (3) if $H$ is a strong hierarchical graph and $V^{\prime} \neq \emptyset$ is a set of vertices with $V(H) \cap V^{\prime}=\emptyset$, the graph $H \otimes V^{\prime}$ is a strong hierarchical graph. A strong hierarchical influence graph is an influence graph $(G, f)$ where $G$ is a strong hierarchical graph.

Theorem 3.4 The Power problem, for oblivious and non-oblivious influence models corresponding to strong hierarchical influence graphs, is polynomial time solvable.

Proof. [Sketch] Let $\mathcal{M}=\mathcal{M}(V, D, q)$ be the oblivious or the non-oblivious model corresponding to a strong hierarchical influence graph $(G, f)$. Fix a participant $i$. For $x \in\{0,1\}^{n}$ let $x^{\prime}=\bar{x}_{-i}, y=D(x)$ and $y^{\prime}=D\left(x^{\prime}\right)$. Consider a table $T(a, b, c, d), 0 \leq a, c \leq n$ and $0 \leq b, d \leq|\mathrm{FI}(G)|$ holding the following quantities

$$
\begin{aligned}
\mid\left\{x \in\{0,1\}^{n}\right. & ||\{j \mid D(x)=1\}|=a \wedge| F(X(x) \cap N) \cap \mathrm{FI}(H) \mid=b \\
& \left.\wedge\left|\left\{j \mid D\left(x^{\prime}\right)=1\right\}\right|=c \wedge\left|F\left(X\left(x^{\prime}\right) \cap N\right) \cap \mathrm{FI}(G)\right|=d\right\} \mid .
\end{aligned}
$$

Observe that

$$
\operatorname{Pow}(i)=\sum_{a \geq q, c<q} \sum_{0 \leq b, d \leq \mathrm{FI}(H)} T(a, b, c, d) .
$$

The values in the table $T$ can be computed recursively, using the hierarchical structure of $G$, from the tables corresponding to different subgraphs of $G$. However, the recurrences are different for the oblivious than for the nonoblivious model. Our algorithms use dynamic programming to compute the table $T$ in polynomial time.

In a star influence graph, in addition to the sets L, I and F, we have the central node $c$ wich acts as mediator and the set $\mathrm{R}$ of reciprocal actors.

Definition 3.5 A star influence graph is an influence graph $(G, f)$, where $V(G)=\mathrm{L} \cup \mathrm{I} \cup \mathrm{R} \cup\{c\} \cup \mathrm{F}$ and $E(G)=\{(u, c) \mid u \in \mathrm{L} \cup \mathrm{R}\} \cup\{(c, v) \mid v \in \mathrm{R} \cup \mathrm{F}\}$. As usual in a star influence model, $\mathcal{M}(G, f, q, N)$, we take $N=\mathrm{L} \cup \mathrm{R} \cup \mathrm{I}$. 
Without loss of generality we can assume that the labeling function of a star influence graph satisfies $f(i) \in\{0,1\}$, for $i \in V(G) \backslash\{c\}$.

Theorem 3.6 The Power problem, for oblivious and non-oblivious models corresponding to star influence games, is polynomial time solvable.

Proof. [Sketch] Let $\mathcal{M}=\mathcal{M}(V, D, q)$ be the oblivious model corresponding to a star influence influence graph $(G, f)$ with center $c$. Fix a participant $i$. For $x \in\{0,1\}^{n}$ let $x^{\prime}=\bar{x}_{-i}$. In the oblivious model we know that when $p_{c}^{1}(x) \geq k$ and $p_{c}^{1}\left(x^{\prime}\right) \geq k$ or when $p_{c}^{1}(x)<k$ and $p_{c}^{1}\left(x^{\prime}\right)<k C_{\mathcal{M}}(x)=C_{\mathcal{M}}\left(x^{\prime}\right)$ and thus $i$ has no power to change the collective decision by changing its initial decision. We analyze the case $p_{c}^{1}(x) \geq k$ and $p_{c}^{1}\left(x^{\prime}\right)<k$ depending on whether $i$ is a leader, a follower, $c$ or a reciprocal actor. In all the cases we can provide a characterization of the set of initial decision vectors in which player $i$ has the power to change the final decision according to its initial decision. Those characterizations allows us to count the number of their elements and thus to compute $\operatorname{Pow}_{\mathcal{M}}(i)$. For the non-oblivious model the proof follows the same lines, however we have to take into consideration further cases in particular those in which $c$ is convinced to change an initial 1 into a 0 which never happens in the oblivious model.

Finally, we want to point out that the complexity of SATISFACTiOn and Power for OLF models remains open as well as the complexity of ExPANSION for two layered bipartite graphs under the simple majority rule.

\section{References}

[1] P. F. Lazarsfeld, B. Berelson, and H. Gaudet. The people's choice - How the voter makes up his mind in a presidential campaign. Columbia University Press, New York, NY, 3rd edition, 1968. First edition published in 1944.

[2] X. Molinero, F. Riquelme, and M. Serna. Measuring satisfaction in societies with opinion leaders and mediators. Corr, abs/1605.04399, 2016.

[3] X. Molinero, F. Riquelme, and M. J. Serna. Cooperation through social influence. European Journal of Operation Research, 242(3):960-974, 2015.

[4] R. van den Brink, A. Rusinowska, and F. Steffen. Measuring power and satisfaction in societies with opinion leaders: dictator and opinion leader properties. Homo Oeconomicus, 28(1-2):161-185, 2011. 